\title{
Enablers and Barriers to Implement COVID-19 Measures in Long-term Care Facilities: A Mixed Methods Implementation Science Assessment in Chile
}

Jorge Browne Salas

Pontificia Universidad Catolica de Chile

Josefa Palacios ( $\square$ mjpalaci@uc.cl)

Pontificia Universidad Católica de Chile: Pontificia Universidad Catolica de Chile

https://orcid.org/0000-0001-6450-971X

Pablo Villalobos Dintrans

Universidad de Santiago de Chile

Ignacio Madero-Cabib

Pontificia Universidad Catolica de Chile

Rocio Quilodran

Pontificia Universidad Catolica de Chile

Alejandro Ceriani

Pontificia Universidad Catolica de Chile

Danilo Meza

Pontificia Universidad Catolica de Chile

\section{Research}

Keywords: COVID-19, nursing homes, long-term care, implementation science

Posted Date: December 11th, 2020

DOI: https://doi.org/10.21203/rs.3.rs-125014/v1

License: (c) (i) This work is licensed under a Creative Commons Attribution 4.0 International License. Read Full License 


\section{Abstract}

Background

The COVID-19 affected disproportionately older people, and particularly people living in long-term care facilities. Considering this problem, the Chilean government issued a series of guidelines and protocols to prevent and manage COVID-19 outbreaks in these facilities.

Methods

This study aims to identify barriers and enablers that affect the implementation of these prevention and management measures. For the analysis, we used an implementation science approach and a mixedmethod strategy-survey to facilities' managers and interviews to carers-, classifying enablers and barriers into four categories: agreement with the intervention's goals, financial resources to implement the measures, technical needs of the intervention, and cultural factors in the facilities.

Results

Results highlight the importance of the four aforementioned factors in the implementation of COVID-19 guidelines and protocols. Managers and caregivers differ in their view of the main enablers and barriers for implementation. However, they both identify the knowledge about the measures and availability of personal protective equipment as enablers and human resources as a potential barrier.

Conclusions

The identification of several factors related to goals and culture highlights the need to adopt a broad implementation approach when designing intervention for long-term care facilities, avoiding restricting the discussion to resources availability.

\section{Contributions To The Literature}

- The article highlights the need to adopt an implementation science perspective for designing, implementing, monitoring and evaluating intervention targeted to older people.

- It presents useful information for policy makers on a high-relevance problem, as COVID-19 in longterm care facilities.

- Results show the relevance of considering usually ignored aspects of implementation, such as alignment of goals and cultural issues.

- The use of a mixed method approach helped not only assess the success of the intervention but also identify relevant implementation factors, key for redesign future policies.

\section{Introduction}


Older people (OP) living in long-term care facilities (LTCF) have been disproportionately affected by COVID-19. Early epidemiological studies described lethality rates that could reach up to $35 \%$ within these facilities (McMichael et al 2020). Currently, evidence suggests that COVID-19-related deaths in LTCF represent $46 \%$ of total COVID-19-related deaths, exceeding $80 \%$ in some countries (Comas-Herrera et al 2020a). To minimize the impact of COVID-19 in LTCFs, the World Health Organization (WHO) has called for including LTCF in each country's response and mobilizing funding to implement prevention and management strategies in these facilities (WHO, 2020); accordingly, several countries have designed and implemented LTCF-specific prevention and management measures (Comas-Herrera et al 2020b).

In Chile, policy responses to tackle COVID-19 in LTCF included banning visitors and implementing sanitary barriers in each facility (Ministerio de Salud, 2020). Furthermore, the Ministry of Health $(\mathrm{MoH})$, the National Service for Older People (Servicio Nacional de Personas Mayores, SENAMA) and the Chilean Geriatrics and Gerontology Society (SGGCh) generated a set of enforceable and unenforceable prevention and management measures, which included:

- Use Personal Protective Equipment (PPE)

- Cleaning and disinfection (including clothes)

- Implementing isolation areas

- Implementing a clean area for staff

- Actions needed when a COVID-19 case is suspected

- Instructions to manage COVID-19-related or non-related deaths in the residence

- Information regarding other mitigation strategies such as transfer to sanitary houses

Furthermore, SENAMA implemented a mitigation strategy based on face-to-face technical support, PPE supply, staff replacement, field testing with rt-PCR and the temporary transfer of COVID-19 residents to sanitary houses (Browne et al., 2020). These measures were summarized in a series of guidelines and protocols for COVID-19 prevention and management, issued by the government in April 2020 (SENAMA 2020).

Although, these initiatives follow international recommendation and best practice observed in other countries (Comas-Herrera et al 2020b), an adequate design of prevention and management measures contributes to, but does not necessarily ensure, their effectiveness. Other factors related to the implementation process could play a critical role. For example, in the UK Rajan and McKee (2020) describe that working partnership with authorities, staff morale and wellbeing, and PPE supply, were critical factors that enabled adequate implementation of preventive measures.

In Chile, informal carers provide care for the great majority of older persons with support needs (Villalobos Dintrans, 2019; Palacios et al., 2020). Institutional facilities provide care to approximately 25,000 older persons ( $1.4 \%$ of the population 65 or older) in a mix of public, private non-profit, and private for-profit institutions that mainly operate nursing-home facilities (Villalobos Dintrans, 2018; Browne et al., 2020). These facilities require the authorization of the $\mathrm{MoH}$, which regulates infrastructure (e.g., number 
of allowed residents per room) and human resources (e.g., number of carers per resident). Healthcare processes, such care in isolation areas and other infection-disease-control measures are not regulated nor surveyed (Ministerio de Salud 2010). Therefore, a healthcare-oriented organizational culture is not necessarily rooted in these facilities. These factors can, in turn, pose barriers for the implementation and the effectiveness of the COVID-19 prevention and management strategy.

This study aims to identify barriers and enablers that affected the implementation of prevention and management of the COVID-19 measures designed by Chilean policymakers for LTFCs. We draw on an implementation science framework, looking at organizational-level factors that explain differences between the results intended by policymakers and observed results. Data was collected and analyzed using a mixed-method strategy (on-line survey and in-depth semi-structured interviews) applied on the two largest non-profit LTFC providers in Chile.

\section{Materials And Methods}

\section{Framework of analysis: Implementation science}

Implementation science (IS) helps understanding and translating theoretical interventions and scientific knowledge into practice in a real-world setting (Villalobos Dintrans et al., 2019). This approach acknowledges the difference between efficacy (outcome of an intervention under ideal conditions) and effectiveness (outcome of an intervention under normal conditions) when translating evidence-based research into practice in the real world, the latest being the focus of the IS analysis (Steckler and McLeroy, 2008; Damschroder and Hagedorn, 2011; Spiegelman, 2016). This distinction is important from a theoretical perspective but particularly relevant when designing, implementing and evaluating public policies. Several IS models and frameworks exist today, many of them applied to health interventions (Chaudoir et al., 2013; Moullin et al., 2015). A common element in these models is the identification of key implementation elements, which usually correspond to features of the intervention provider, the intervention, the recipient, and the environment (Villalobos Dintrans et al., 2019).

This study focuses on identifying enablers and barriers for the implementation of national-level COVID-19 guidelines and protocols in LTCF. Following Villalobos Dintrans and Bossert (2017), we consider four implementation factors:

i. Goals: whether the intended results and mechanisms of the provider and recipient are aligned, and if they are aligned with the intervention's objective.

ii. Financial factors: whether resource constraints can explain the ability to implement the intervention as intended.

iii. Technical requirements: whether the technical needs of the intervention match the technical capacities of the institutions involved in the project.

iv. Culture: whether the usual way to carry on the activities in the institutions conflicts with the proposed changes. 
Figure 1 shows the framework we used to identify enablers and barriers for implementation. We focused on the interaction between two implementation elements (in blue): intervention and recipient (Chambers et al., 2013), looking at the four implementation factors (in red). The intervention in this case is the official COVID-19 guidelines and protocols issued by the government, as described in SENAMA (2020). The intervention providers are the Ministry of Health and the National Elderly Office. The recipients of the intervention-the target population-are authorized LTCF in the country.

\section{Study design}

We draw on a mixed-method design based both on a quantitative survey to managers of LTCF and qualitative semi-structured interviews to LTCF carers. The quantitative data to managers allowed us to contextualize findings, assess the implementation degree of the COVID-19 measures (e.g., staff replacement), and to compare enablers and barriers (e.g., PPE availability) across different institutions and levels. The qualitative data allows us to identify previously unknown organizational-level enablers and barriers among the ultimate implementers (i.e., the carers).

\section{Data collection}

Sample

While the recipient of the intervention-LTCF-is clearly identified, two different actors, LTCF's managers and carers, implement the government's intervention within each facility.

We sample managers and carers from LTCF in three regions of the country (Metropolitana, Libertador General Bernardo O'Higgins, and Valparaíso). Selected LTCF belong to "Hogar de Cristo" and "Fundación las Rosas" the two largest non-profit LTCF providers in Chile. Together, these providers run 41 nursing homes with 2,770 residents, accounting for almost $30 \%$ of all residents in non-profit institutions in the country. All of "Hogar de Cristo" and "Fundación las Rosas" facilities have health professionals (a nurse, physiotherapist and/or physician) and independent technical support on location.

We sent a quantitative online survey to all of these LTCF's managers $(n=41)$. Additionally, we selected four of these LTCFs and conducted two semi-structured interviews with carers on each one $(n=8)$. This subsample was purposely selected to cover LTCFs in the three regions, as well as facilities with COVID-19 outbreaks: at the time of interview, two LTCFs had past and two had ongoing COVID-19 outbreaks; two LTCFs were drawn from the Región Metropolitana, one from Valparaiso and one from Libertador General Bernardo O'Higgins. In each of the four selected LTCF, the administrator provided the contact of the carers to be interviewed. The sample was not designed to be representative of LTCF carers but to cover the variability in the experiences of the population of LTCF carers (Patton, 2002). All participating carers are women; all but one of the participating carers are Chilean. 
Survey questions probed the barriers and enablers using the previously described implementation factors (goals, financial factors, technical requirements, and culture). The survey was divided into two parts: one asking about prevention measures and other with questions on control measures, both parts had the exact same questions (see Supplementary Material 1). The survey was carried out between July 10-21, 2020. The response rate was $88 \%$ (36 out of 41 ).

The eight semi-structured interviews were also carried out during July 2020. Interview questions followed the same guideline to assess barriers along the four IS factors (see Supplementary material 2). Five of the study authors carried out the interviews by phone. Interviews lasted around 40 minutes on average, were audio recorded (under participants' consent) and were transcribed verbatim. We pre-tested the instrument by applying it to a carer in the same conditions planned for the selected sample. The pre-test allowed us to verify that appropriate questions were being asked and that questions did not make respondents uncomfortable and/or confused (Hurst et al., 2015). After each interview, and following standards in qualitative research, each interviewer collected field notes and shared them with the other authors. Notes included a critical reflection on how the details of the interview process and the important issues arising during the conversation (Phillippi \& Lauderdalle, 2018).

\section{Data analysis}

Quantitative data was analyzed with R, while a thematic analysis was used to look at the qualitative data collaboratively (Braun and Clark, 2012). First, all transcriptions and field notes were clustered and analyzed using the four IS factor (goals, financial factors, technical requirements and culture). Then, data was studied within each factor, identifying relevant themes and sub-themes and classified each quote accordingly. Following Graneheim and Lundman (2004), and in order to increase the validity of the analysis, more than one author participated in each stage, discussing results to obtain consensus. We present the qualitative and quantitative findings simultaneously, in accordance with a mixed-methods approach and in recognition of the complementarity of the two approaches.

\section{Results}

Findings are presented according to the four implementations factors, highlighting the enablers and barriers that both managers and carers identify in relation to each factor. As managers' differ in their assessment of implementing prevention and management measures ( $11 \%$ report that implementing prevention measures is not possible, while this figure reached $19 \%$ for control measures), information is are presented separately for each type of measure.

\section{Enablers and barriers to implementation}

We identify different elements within each implementation factor that could be classified as barriers or enablers to implementation (Table 1). Managers and carers share similar perceptions on these barriers and enablers, yet carers and managers provided different insights for the same implementation factor 
(e.g., human capital: quality and quantity). Table 1 summarizes the enablers and barriers identified in each factor, noting whether it was identified by managers (MG) or carers (CR).

\section{Goals}

Regarding the alignment of goals between the provider, intervention, and recipients, the survey shows that $80.6 \%$ and $75.0 \%$ of managers have a high degree of knowledge of the COVID-19 prevention (e.g., PPE use and disinfection) and control (e.g., setting an isolation area) measures, respectively. Most managers report that they agree or strongly agree with the prevention measures $(94.6 \%)$ and control measures $(97.2 \%)$ in the guidelines and protocols.

Similarly, carers perceive that interventions are important and accurate, indicating an adequate alignment between the goals of implementers and the measures proposed. Fear, uncertainty, and the burden of COVID-19 on LTCF residents, are strong incentives to adopt these measures. The fear of contracting the virus acts as an enabler to implement preventive measures. It motivates carers to implement these measures not only while at the LTCF, but also outside in public spaces or even at home:

"We are following these measures from day one, because we don't know if there will be another outbreak. We need to do everything to protect older people and ourselves. The hardest thing is fear. Fear to be infected. Even when using the PPE [personal protective equipment]. Fear overcomes me." (I1)

"The measures are OK. Anything to protect the grandpas is fine." (I2)

Carers value the quick response from authorities regarding measures aimed at LTCF. They also perceive the need to contribute in the implementation of the measures; they acknowledge that it is both their and the clinical team's responsibility to protect the residents and themselves. Carers accept the measures and implement them without questioning whether they are the most effective or efficient measures. They recognize that the measures are designed to protect older people and implement them as ordered.

"We have to believe this [the implementation of the preventive measures established in protocols] is the right way [to prevent and mitigate COVID-19]."

"We need to be careful, because we are the ones that go outside, not them [residents]." (I1)

Notwithstanding the enablers identified above, the origin of the protocols is unknown for carers, and rather seen as a top-down measure. This can act as a barrier to implementation.

"I think it was the nurse in charge of the residency who created the protocols, she made the protocol, and then she taught it to us." (I3)

"I have no idea where they came from [the measures]. Perhaps they were done by the boss or someone else, but honestly I have no idea where they come from." (14) 


\section{Resources}

PPE availability enables implementation. First, in terms of Personal Protective Equipment (PPE) availability, around $20 \%$ of managers perceive PPE shortage $(22.4 \%$ and $25 \%$ for prevention and control measures respectively). Carers share this perception, although some carers reported problems in accessing specific items. Carers also noted an increase in resources during the pandemic:

"We have been given all the PPE we need." (15)

"We changed our aprons every two older adults, because there weren't that many. Now there are more supplies, and we change aprons and gloves." (16)

Only one participant commented that the LTCF did not provide EPP and she had to pay for the equipment out of her own pocket:

"The goggles, those I had to buy them myself." (17)

Infrastructure was a key resource and was rarely capable to meet to the protocols' requirements. Many of the LTCF physical configurations are difficult to adapt to a pandemic situation, as they were not designed to be adaptable to emergencies of this magnitude. Half of the managers reported that their infrastructure was inadequate to implement the measures; carers made a similar assessment:

"We didn't have the infrastructure. As I told you, this is new for everyone." (I3)

"In architectural terms, it is not possible to add more bathrooms." (I5)

The survey shows that the most frequent barrier to implement the measures was staff availability for prevention (55.5\%) and management measures (52.8\%). Carers also identified human capital as one of the crucial issues in dealing with the pandemic and implementing the protocols. For carers, however, the critical issue is quality rather than availability. Though carers acknowledge the need to bring new staff to the LTCF, they criticize the lack of adequate training or experience among the newly recruited staff:

"They sent a lot of people, but they were not prepared to work here. They had no experience." (I3)

Additionally, participants report a lack of technical and psychological support for carers, a key aspect for LTCF staff (WHO, 2020). Carers resent this lack of support as they struggle both with the provision of services and the pandemic on a day-to-day basis, while also dealing with their own fears, and facing the fear and the real possibility of death of the older people they care about:

"I insist. There is a lack of support-psychological, pedagogical, training-for the carers." (I8)

\section{Technical requirements}


In general, participants declared that the guidelines and protocols were easy to understand. Surveyed managers perceive no barriers in understanding the prevention (100\%) and control (96.8\%) measures. However, practically no carers reported having seen the actual protocols (only one did). Carers got the information from posters and relied on LTCF managers and the technical staff indications:

"Our boss, she is a nurse and she knows a lot. We have an advantage there. She anticipates the facts. Something like that." (I2)

Both managers and carers valued the training. Around $60 \%$ of managers felt that staff had adequate training to implement the measures. Carers declared that training was key to know what to do. However, there can be issues with the interpretation and the emphasis on a particular mention when trainers or managers are the sole source of information for prevention and control of COVID-19. As one of the interviewer noted:

"She [carer] listed all the prevention measures but couldn't say which was the most important (e.g. washing hands) and kept repeating others that were not in the guidelines (e.g. shoes sanitization)." (RI)

Another aspect of the technical requirements in implementing the guidelines and protocols is supervision. Carers acknowledge having received training on the protocols but highlight that there was few to null supervision on the actual implementation:

"They ask us to wash our hands, and they give us the equipment... but honestly, there is no much control." (I5)

\section{Culture}

Trust between carers and health professionals working at the LTCF can affect the effectiveness of the intervention:

"The professionals told us there were respiratory problems... but all those were also coronavirus symptoms... we were working with infected people and we didn't know." (I4)

Yet, the workplace environment was generally perceived as positive. Most managers reported that the staff agreed and/or strongly agreed with the prevention (94.4\%) and management (91.7\%) measures. Carers described the job and their colleagues in good terms, reinforcing the idea that they felt part of a group that shared a common goal (esprit de corps):

"We work together in a good working environment; here you feel good... we are a very bonded group and we support each other..." (19)

However, personnel turnover during the outbreaks altered the perception of this good environment generating tension between the newly recruited carers and those with a longer tenure: 
"The new ones. They came here knowing nothing about this..." (13)

"The old carer, she has years of experience and old habits. She has her own way to do the job. And she wants to impose her style, the old way, and wants to pull down the new." (I3)

Finally, carers reported they were able to adapt their everyday life routines to the pandemic measures. However, they acknowledged that both the residents and the carers themselves struggled to comply with some of these measures. There are socio-emotional (e.g., wanting to spend time and share a space with others) as well as practical factors (e.g., barriers for communication) explaining this struggle:

"They [some residents] gather anyway. They want to have lunch together, and we have to separate them." (I5)

"Before, we shared more time with them [residents]. If someone asked for you, you went. Now, we can only see the residents we have assigned." (16)

“They [residents] ask me why I'm wearing a costume. They don't understand why we should use a mask if there is nothing wrong with them. It makes them feel bad." (I5)

"Using the face mask was difficult, because it gives you a feeling of suffocation." (I3).

\section{Discussion}

Most of the previous assessments of LTCF and the implementation of guidelines and protocols in the context of COVID-19 have focused in one specific dimension of the implementation. Usually, studies focus on resources and technical issues (e.g., training). Indeed, staff, infrastructure and PPE availability had been identified as key elements in other settings (WHO, 2020; Comas-Herrera et al., 2020c). However, the research has neglected key dimensions that influence effectiveness, such as the alignment of the measures with the implementer's goals and cultural issues.

Our study shows the relevance of IS to guide and evaluate the implementation of COVID-19 measures on LTCF: having good technical-quality protocols (efficacy) is important but will not ensure results on the field (effectiveness). In adopting a broader framework through IS, this study identified several implementation barriers (e.g., infrastructure, human capital and resistance to chance), and enablers (e.g., PPE availability and trust in technical staff) that could facilitate or hinder the effectiveness of the measures proposed by the central government.

Managers and carers tend to agree on the identification of different barriers and enablers but also report differences. Notably, both managers and carers recognize human capital as critical element for implementation. However, managers identified the availability of staff as a barrier while carers referred more to the quality, noting that the lack of experience and training of newly recruited staff acted as a barrier for implementation. 
The LTCF analyzed here illustrate the relevance of identifying all the elements involved in the intervention. This means acknowledging the existence of two levels in which the intervention is adopted by a LTCF, and the different roles played by managers and carers. Results also emphasize the usefulness of using a mixed-methods approach to analyze the problem, particularly with different actors involved in the process of implementation. On the one hand, through the survey, managers reported their overall perception and this allowed us to quantify and collect standardized information. Through the semi-structured interviews, carers identified several key aspects of the implementation based on their daily experience working at the LTCF.

We acknowledge some limitations of our study. While interpreting the results it is important to consider external validity, which is limited in both scope and time. First, the sample included managers and carers from 41 LTCF owned by two non-profit LTCF providers. Although they are important players in institutional care in Chile, the situation described in these LTCF might be different to those observed in other facilities. Size, type of administration, and institutional culture can vary across providers and influence the results. Second, the analysis is constrained to a specific period of time. The COVID-19 pandemic has shown to be complex and dynamic. Consequently, managers and carers' perceptions are influenced by the moment and experiences lived by the time the survey and the interviews were carried out.

Finally, in terms of policy implications, we extract several lessons. First, we highlight the importance of building networks between the different players involved in the work of LTCF. Peoples' responses show that trust in administrative and technical staff, as well as the previous relationship established between the national and local level institutions, was key for a quick communication and implementation of the guidelines and protocols. Second, we acknowledge again the relevance of designing interventions not only technically correct but also feasible to implement for the intended target population (e.g., resources available and infrastructure requirements). Also, from a psychological perspective, understanding people's resistance to change and their limits in terms of implementing measures that goes against their goals and culture. Third, human capital is a key issue but it is not restricted to staff availability. Having a network of well-trained carers is key, and implies moving towards policies to train but also certify old and new carers. Finally, the analysis was focused on understanding the core elements of the intervention, leaving aside the importance of pre- and post-intervention components (Villalobos Dintrans et al., 2019). The design of new policies benefits from better pre-intervention (e.g. how many LTCF are in the country? Where they are? What are their conditions?) and post-intervention actions (monitoring and evaluation of the measures) in order to generate effective improvement to LTCF residents and their staff.

\section{Conclusions}

The results highlight the usefulness of using a IS approach to design and evaluate the implementation of interventions in LTCF. The study uses the COVID-19 measures in Chile as an example but the recommendation can be generalized to design and evaluate intervention in different contexts. 


\section{Abbreviations}

CR: carers

IS: Implementation Science

LTCF: long-term care facilities

MG: managers

MoH: Ministry of Health

OP: Older people

PPE: Personal Protective Equipment

SENAMA: National Service for Older People (Servicio Nacional de Personas Mayores)

SGGCh: Chilean Geriatrics and Gerontology Society

WHO: World Health Organization

\section{Declarations}

\section{Ethics approval and consent to participate}

The Ethics Review Board of the Pontifica Universidad Católica de Chile approved the study design, instruments and methods (Res.\# 012321).

\section{Consent for publication}

Not applicable

\section{Availability of data and materials}

The datasets and interviews used and analysed during the current study are available from the corresponding author on reasonable request.

\section{Competing interests}

The authors declare that they have no competing interests

\section{Funding}

This work was supported by the Sección de Geriatría, Facultad de Medicina, Pontificia Universidad Católica de Chile and by the following grants: 
ANID/Millennium Science Initiative Grant "Millennium Nucleus for the Study of the Life Course and Vulnerability (MLIV)" NCS17_062

ANID/FONDAP/N¹5130009

ANID/FONDECYT/INICIACION/Nº11180360

\section{Authors' contributions}

$R Q, A C, D M, J P$ designed the interview guidelines, recruited participants and collected and analysed the qualitative data. JB, IM-C, PV designed the survey instrument, sample and data collection procedures, and analysed the quantitative data. JP, PV and JB drafted the manuscript. All authors read, commented and approved the final manuscript

\section{Acknowledgements}

Not applicable

\section{References}

Braun V, Clarke V. Thematic analysis. APA handbook of research methods in psychology. 2012; 2:57 -71.

Browne J, Fasce G, Pineda I, Villalobos P. Policy responses to COVID-19 in Long-Term Care facilities in Chile [Internet]. LTCcovid.org, International Long-Term Care Policy Network, CPEC-LSE; 2020. Available in https://ltccovid.org/wp-content/uploads/2020/07/The-COVID-19-Long-Term-Care-situation-in-Chile-24July-2020-3.pdf

Chaudoir SR, Dugan AG, Barr CHI. Measuring factors affecting implementation of health innovations: a systematic review of structural, organizational, provider, patient, and innovation level measures. Implementation Science [Internet] 2013; 8: 22. Available in https://www.ncbi.nlm.nih.gov/pmc/articles/PMC3598720/pdf/1748-5908-8-22.pdf

Chambers DA, Glasgow RE, Stange KC. Implementation Science [Internet] 2013; 2:117. Available in https://implementationscience.biomedcentral.com/articles/10.1186/1748-5908-8-117

Comas-Herrera A, Zalakaín J, Lemmon E, Henderson D, Litwin C, Hsu AT, Schmidt AE, Arling G and Fernández J-L. Mortality associated with COVID-19 in care homes: international evidence [Internet]. LTCcovid.org, International Long-Term Care Policy Network, CPEC-LSE; 2020. Available in: https://ltccovid.org/2020/04/12/mortality-associated-with-covid-19-outbreaks-in-care-homes-earlyinternational-evidence/

Comas-Herrera A, Ashcroft E, Lorenz-Dant K. International examples of measures to prevent and manage COVID-19 outbreaks in residential care and nursing home settings [Internet]. LTCcovid.org, International Long-Term Care Policy Network, CPEC-LSE; 2020. Available in https://tccovid.org/2020/05/02/new- 
report-international-examples-of-measures-to-prevent-and-manage-covid-19-outbreaks-in-residential-careand-nursing-home-settings/

Comas-Herrera A, Ashcroft E, Lorenz-Dant K. International examples of measures to prevent and manage COVID-19 outbreaks in residential care and nursing home settings [Internet]. LTCcovid.org, International Long-Term Care Policy Network, CPEC-LSE; 2020. Available in https://ltccovid.org/wpcontent/uploads/2020/05/International-measures-to-prevent-and-manage-COVID19-infections-in-carehomes-11-May-2.pdf

Damschroder LJ, Hagedorn HJ. A guiding framework and approach for implementation research in substance use disorders treatment. Psychology of Addictive Behaviors. 2011; 25(2): 194 - 205.

Graneheim UH, Lundman B. Qualitative content analysis in nursing research: concepts, procedures and measures to achieve trustworthiness. Nurse Education Today. 2004; 24(2):105-112.

Hurst S, Arulogun OS, Owolabi MO, Akinyemi R, Uvere E, Warth S, Ovbiagele B. Pretesting qualitative data collection procedures to facilitate methodological adherence and team building in Nigeria. International journal of qualitative methods. 2015; 14(1): $53-64$.

McMichael TM, Currie DW, Clark S, et al. Epidemiology of Covid-19 in a Long-Term Care Facility in King County, Washington. N Engl J Med [Internet] 2020: 2005 - 2011. Available in https://www.nejm.org/doi/pdf/10.1056/NEJMoa2005412?articleTools=true

Ministerio de Salud. Aprueba reglamento de establecimientos de larga estadía para adultos mayores [Internet]. Biblioteca del Congreso Nacional de Chile; 2010. Available in https://www.minsal.cl/sites/default/files/files/DTO-14_05-AGO-2010_ELEAM.pdf

Ministerio de Salud. Resolución 236 Exenta [Internet]. Biblioteca del Congreso Nacional de Chile; 2020. Available in https://www.bcn.cl/leychile/navegar?idNorma=1144055

Moullin JC, Sabater-Hernández D, Fernandez-Llimos F, Benrimoj SI. A systematic review of implementation frameworks of innovations in healthcare and resulting generic implementation framework. Health Res Policy Syst. 2015; 13(16): 1 - 11.

Palacios J, Pérez P, Webb A. The experience of caring for an older relative in Chile: Going beyond the burden of care. Ageing and Society [Internet] 2020: 1- 20. Available in https://doi.org/10.1017/S0144686X20001567

Patton MQ. Two decades of developments in qualitative inquiry: A personal, experiential perspective. Qualitative social work. 2002; 1(3): $261-283$.

Phillippi J, Lauderdale J. A guide to field notes for qualitative research: Context and conversation. Qualitative health research. 2018; 28(3):381-388. 
Rajan S, Mckee M. Learning from the impacts of COVID-19 on care homes: a pilot survey [Internet]. LTCcovid, International Long-Term Care Policy Network, CPEC-LSE; 2020. Available in https://ltccovid.org/2020/06/09/learning-from-the-impacts-of-covid-19-on-care-homes-in-england-a-pilotsurvey/

Servicio Nacional del Adulto Mayor [SENAMA]. Guía práctica de manejo COVID-19 en establecimientos de larga estadía para adultos mayores (ELEAM) [Internet]. ACHS; 2020. Available in

http://www.senama.gob.cl/storage/docs/Guia_Directivos_ELEAM.pdf

Steckler A, McLeroy KR. The Importance of External Validity. American Journal of Public Health. 2008; 98(1):9-10.

Spiegelman D. Evaluating public health interventions: examples, definitions, and a personal note. American Journal of Public Health. 2016; 106(1):70-73.

Villalobos Dintrans P. Do long-term care services match population needs? A spatial analysis of nursing homes in Chile. PLoS ONE [Internet] 2018; 13(6): 1 - 15. Available in https://doi.org/10.1371/journal.pone.0199522

Villalobos Dintrans P. Informal caregivers in Chile: the equity dimension of an invisible problem. Health Policy and Planning [Internet] 2019; 34: 792-799. Available in https://doi.org/10.1093/heapol/czz120

Villalobos Dintrans P, Bossert TJ. Institutionalization and sustainability of donor-funded quality assurance initiatives: The case of Honduras. Research and Evaluation Report [Internet]. Research and Evaluation Report. Chevy Chase, MD: University Research Co., LLC (URC); 2017. Available in https://www.researchgate.net/publication/326343738_Institutionalization_and_sustainability_of_donorfunded_quality_assurance_initiatives_The_case_of_Honduras

Villalobos Dintrans P, Bossert TJ, Sherry J, Kruk ME. A synthesis of implementation science frameworks and application to global health gaps. Global Health Research and Policy [Internet] 2019; 4: 25. Available in https://doi.org/10.1186/s41256-019-0115-1

World Health Organization [WHO]. Preventing and managing COVID-19 across long-term care services: policy brief [Internet]. World Health Organization; 2020. Available in https://www.who.int/publications/i/item/WHO-2019-nCoV-Policy_Brief-Long-term_Care-2020.1

\section{Tables}

Table 1. Summary of enablers and barriers for the implementation of the COVID-19 guidelines and protocols in LTCF in Chile. 


\begin{tabular}{|c|c|c|}
\hline $\begin{array}{l}\text { Implementation } \\
\text { factors }\end{array}$ & Enablers & Barriers \\
\hline \multirow[t]{3}{*}{ Goals } & Knowledge of measures [MG, CR] & Top-down initiative [CR] \\
\hline & Fear and uncertainty [CR] & \\
\hline & $\begin{array}{l}\text { Common purpose: concern for older } \\
\text { people [CR] }\end{array}$ & \\
\hline \multirow[t]{4}{*}{ Resources } & PPE availability [MG, CR] & Infrastructure \\
\hline & Human capital (quantity) [CR] & Human capital (quality) [CR] \\
\hline & & Human capital (quantity) [MG] \\
\hline & & Support to cares [CR] \\
\hline \multirow[t]{3}{*}{$\begin{array}{l}\text { Technical } \\
\text { requirements }\end{array}$} & $\begin{array}{l}\text { Message clear and easy to } \\
\text { understand [MG, CR] }\end{array}$ & $\begin{array}{l}\text { Lack of first-hand information, } \\
\text { interpretation [CR] }\end{array}$ \\
\hline & $\begin{array}{l}\text { Trust in LTCF administration/ } \\
\text { technical staff [MG, CR] }\end{array}$ & Lack of supervision [CR] \\
\hline & Availability of posters [CR] & \\
\hline \multirow[t]{2}{*}{ Culture } & Esprit de corps [CR] & $\begin{array}{l}\text { Trust issues between different groups } \\
\text { [CR] }\end{array}$ \\
\hline & & $\begin{array}{l}\text { Resistance to change: emotional and } \\
\text { practical factors [CR] }\end{array}$ \\
\hline
\end{tabular}

Note: MG: managers; CR: carers.

\section{Figures}




\section{Implementation elements}
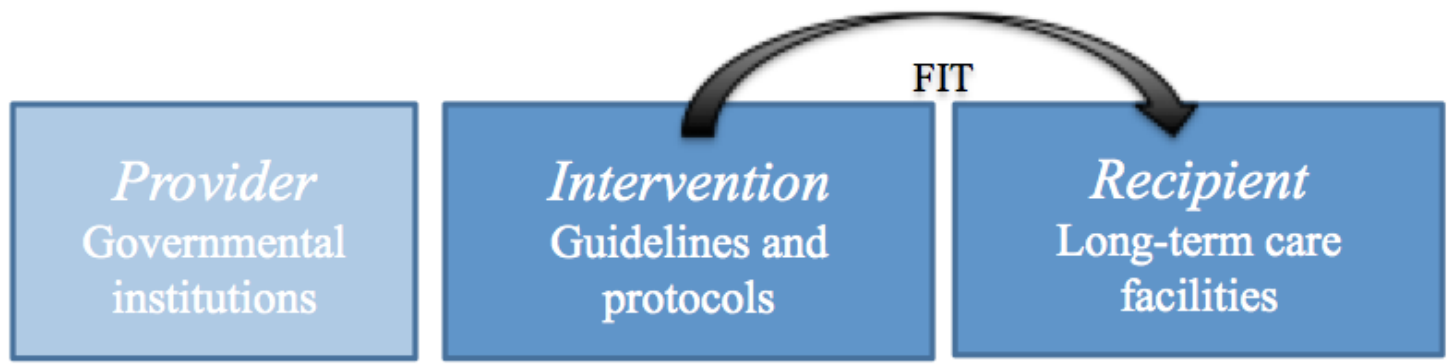

Enviromment

COVID-19

LTCF pre-COVID

situation

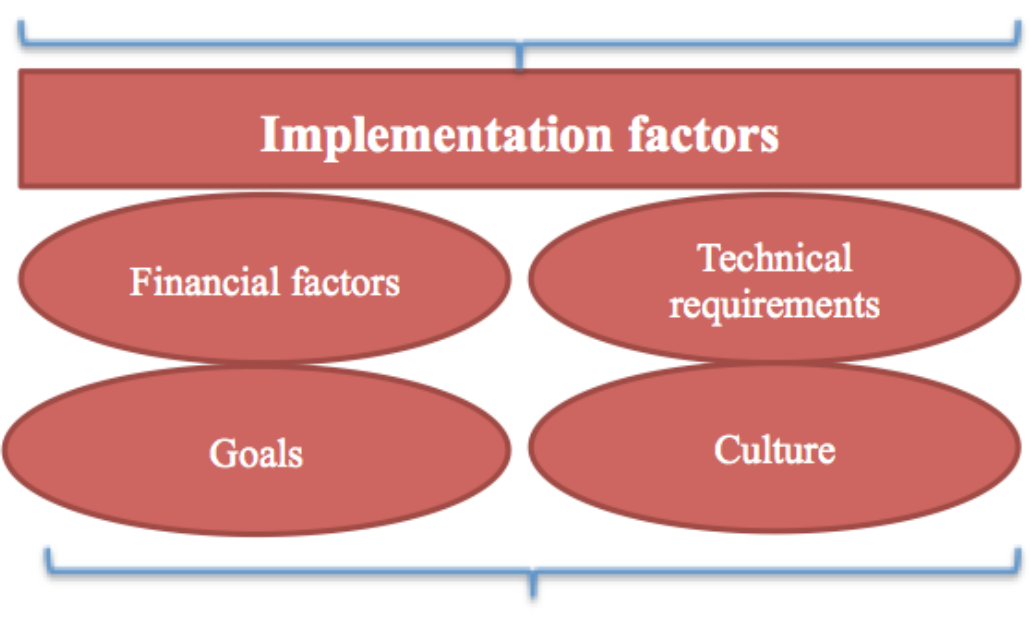

Enablers and barriers for the implementation

\section{Figure 1}

Implementation elements and factors considered in the study

\section{Supplementary Files}

This is a list of supplementary files associated with this preprint. Click to download.

- SupplementaryMaterial1.docx

- Supplementarymaterial2.docx 